\title{
IN-PLANE MODE RESONANT CANTILEVERS AS LIQUID-PHASE CHEMICAL SENSORS WITH PPB RANGE LIMITS OF DETECTION \\ L.A. Beardslee ${ }^{1}$, K.S. Demirci ${ }^{1}$, Y. Luzinova ${ }^{1}$, J.J. Su ${ }^{1}$, B. Mizaikoff ${ }^{3}$, S. Heinrich ${ }^{2}$, F. Josse ${ }^{2}$, O. Brand ${ }^{1}$ \\ ${ }^{1}$ Georgia Institute of Technology, Atlanta, Georgia, USA \\ ${ }^{2}$ Marquette University, Milwaukee, Wisconsin, USA \\ ${ }^{3}$ University of Ulm, Ulm, Germany
}

\begin{abstract}
To reduce fluid damping in liquids and improve the detection limit in liquid-phase chemical sensing applications, resonant cantilever sensors vibrating in their in-plane rather than out-of-plane resonant mode have been fabricated and tested, resulting in measured Q-factors up to 70 in water (up to 4,000 in air) and detection limits in the ppb range for polymer-coated cantilevers exposed to volatile organics in water. In-plane vibrations were thermally excited and detected using piezoresistors arranged in a Wheatstone bridge configuration. The special U-shaped layout of the piezoresistive Wheatstone bridge based on diffused silicon resistors ensures an output signal for the in-plane modes, but suppresses out-of-plane modes, thus enabling stable closed-loop operation of the resonant sensors at the desired first in-plane mode's frequency.
\end{abstract}

\section{INTRODUCTION}

The current state of the art for detecting trace contaminants in water involves field sampling and the use of laboratory instruments, such as liquid and gas chromatography, mass spectrometry, or optical spectroscopic techniques for analysis. Generally, these technologies are not appropriate for in-field use or use by first responders because of their cost and complexity of operation. However, miniaturized versions of such laboratory equipment, in particular micro gas chromatography ( $\mu \mathrm{GC})$ systems [1] and fiber-based IR sensors [2] have made substantial progress in recent years. Both of these technologies show promise for analyzing complex samples in the field, yet both systems are still fairly complex. For targeted detection of a few analytes of interest in a fluid sample, microfabricated chemical sensors and in particular sensor arrays can offer a simple, low-cost alternative. Among possible sensing schemes, mass-sensitive transducers, which sense the analyte mass, have gained interest in recent years. Cantilever-based sensors $[3,4]$ are of particular interest because of their ease of manufacture, and thus are considered here.

Traditionally, cantilevers have been used in either their torsional or out-of-plane flexural modes for resonant sensing applications. The main advantage of using the out-of-plane mode is that it is the lowest-frequency cantilever mode (assuming that the cantilever width is larger than the cantilever thickness) and, thus, is generally the easiest to excite and detect. Resonant silicon cantilevers vibrating in an out-of-plane flexural mode have been successfully employed for chemical sensing in air $[5,6]$ with the cantilevers typically exhibiting quality factors (Q-factors) around 1,000 in air. The dependence of the Q-factor (measured in air) of the fundamental out-of-plane flexural mode on the cantilever geometry has been investigated in detail in [7].

However, in case of liquid operation, the out-of-plane mode is less desirable because of considerable (i) fluid damping (and associated low quality factors) and (ii) mass loading due to the acceleration of the surrounding liquid. Experimental data show quality factors in liquid to be typically around 10 and a reduction of the resonance frequency from the "in-air" to the "in-water" value of up to $50 \%$ [8]. For mass-sensitive (resonant) chemical sensors, the limit of detection is typically defined as three times the ratio of the short-term frequency stability, i.e. the minimal resolvable frequency change, and the sensor sensitivity. While the reduced Q-factor directly impacts the frequency stability, the added fluid mass generally reduces the sensor sensitivity, and it is thus challenging to build robust cantilever-based mass-sensitive chemical sensors with low detection limits for liquid operation.

One promising approach to mitigate damping from fluids in liquid sensing applications has been demonstrated in [9] by creating a fluid channel inside the resonator itself, so that the device can be operated in air or even vacuum, gives considerably improved quality factors compared to liquid operation. The disadvantage of this implementation is both the associated more complex fabrication sequence, and the fact that the resonators with embedded fluid channels currently rely on an external detection scheme using optical measurement setups that do not readily lend themselves to embedded applications. A second approach involves using shear wave acoustic devices, such as the well-known quartz crystal microbalance. While the use of such acoustic wave devices typically yields excellent limits of detection [10], they rely on piezoelectric transducers and their fabrication is typically not compatible with integrated circuit processes.

In the present work, we explore the use of the first in-plane flexural mode of a simple cantilever to address the discussed challenges of liquid-phase chemical sensing using a resonant sensor platform and demonstrate ppb-range detection limits for volatile organic compounds (VOC) in water. Thereby, the improved detection limit stems from both the lower mass loading by the fluid and the improved quality factors in liquid. In addition, mechanical characterization data is presented, which highlights the higher quality factors and also reduced mass loading effects found for these cantilevers.

\section{CANTILEVER DESIGN AND FABRICATION}

Cantilevers with widths ranging from $45 \mu \mathrm{m}$ to $90 \mu \mathrm{m}$ and lengths ranging from $200 \mu \mathrm{m}$ to $1000 \mu \mathrm{m}$ were fabricated and characterized (see Figure 1a). The devices were fabricated and released using a CMOS-compatible bulk micromachining process that is presented elsewhere [11]. The cantilevers feature integrated resistive structures for electrothermal excitation and piezoresistive detection of the first in-plane flexural mode (see Figure 1b). Thermal excitation is favored for liquid operation because it can generate large actuation forces, which are needed to overcome the considerable viscous drag of the surrounding fluid.

A Wheatstone bridge configuration with the four piezoresistors in a U-shaped layout is chosen for vibration detection. Thereby, the placement of the resistors allows for suppression of both the thermal signal from the excitation resistors and also the signals created by torsional and out-of-plane flexural modes. This way, the first in-plane flexural mode generates the strongest detection signal, even though its resonance frequency is several times higher than that of the first out-of-plane mode for the device dimensions investigated in this work. The excitation 
resistors are located close to the clamped edge of the cantilever to minimize the static temperature elevation of the cantilever during operation. The excitation resistors run along the cantilever side to maximize the resulting bending moment. During normal device operation only one of the two resistors is powered with an ac voltage superimposed on a dc bias voltage.

(a)
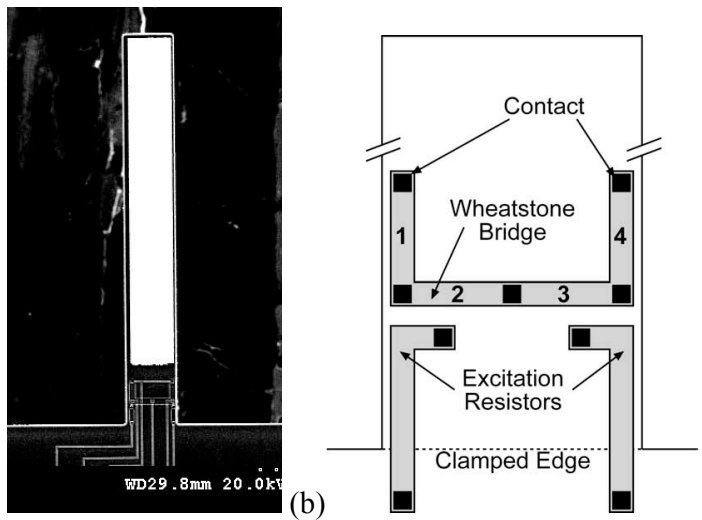

Figure 1: (a) SEM photograph of $60 \mu \mathrm{m}$ wide and $400 \mu \mathrm{m}$ long cantilever (coated with gold for biochemical sensing applications); (b) schematic layout of heating resistors and U-shaped piezoresistive Wheatstone bridge.

(a)

\section{In-Plane Flexural Mode}

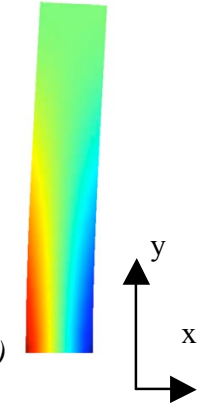

(b)

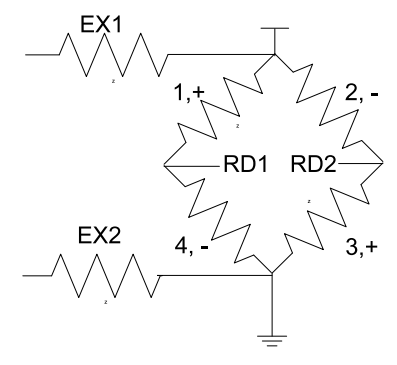

Out-Of-Plane Flexural Mode

(a)

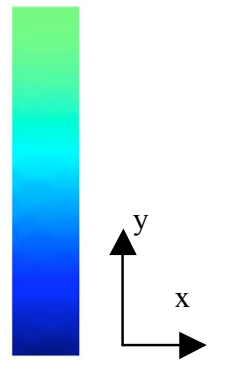

(b)

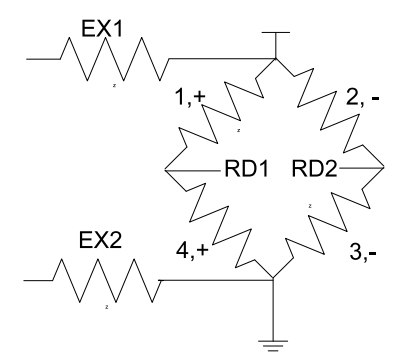

Figure 2: (a) Distribution of the bending stress component $\sigma_{y}$ in $y$-direction as simulated using the FEM software COMSOL for a $75 \mu \mathrm{m}$ wide and $400 \mu \mathrm{m}$ long cantilever vibrating in the first out-of-plane and the first in-plane resonance mode; (b) Signs of the expected resistance change of the piezoresistors (see Fig. 1) in case of the first out-of-plane and the first in-plane mode, assuming that resistor 1 experiences a positive resistance change. RD1 and RD2 represent the bridge output terminals, and EX1 and EX2 denote the two excitation resistors.
The resistor placements were chosen by examining the symmetry of the longitudinal stress component in the cantilever's flexural vibration modes. Figure 2 compares the simulated stress on the cantilever surface in y-direction, i.e. along the cantilever length, of the first in-plane and the first out-of-plane flexural mode. The resistors were placed and connected in a way that the resistance changes associated with the in-plane mode result in a bridge output signal, while the resistance changes associated with the out-of-plane mode yield a largely suppressed output signal. Using p-type piezoresistors aligned along the [110] directions, the longitudinal and transverse piezoresistive coefficients have similar magnitude but opposite sign [12], and the in-/out-of-plane flexural mode shapes result in the characteristic resistance changes highlighted in Figure 2.

\section{SENSOR PACKAGING}

For liquid testing the resonators were packaged using acrylic manifolds fabricated on a stereolithography tool (see Figure 3). The manifolds were glued into 28-pin DIL (Dual-In-Line) packages, which allows for electrical connection to the die via wire-bonding. An acrylic ring was glued to the surface of the die, and silicone gaskets were used to seal the bottom of the die to the lower manifold and to seal the top manifold (which screws into the thermoplastic inserts on the DIL package) to the glued-on ring, thus creating a flow cell. For chemical sensing experiments the flow cell allows a sample to flow from top to bottom through the micromachined opening in the sensor chip. For mechanical characterization in liquid, a drop of water was simply placed on the area enclosed by the ring and allowed to percolate through the bulk micromachined opening, immersing the cantilever.

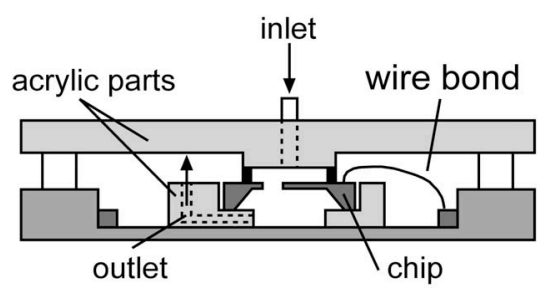

Figure 3: Schematic cross-section of packaging concept with fluid flow through the etch opening in the sensor chip (top to bottom).

For chemical measurements a thicker surface protection was needed to protect the aluminum lines on the device surface from corrosion during liquid operation. The passivation used in this work consisted of three layers of pulse-deposited PECVD silicon oxide followed by PECVD silicon nitride. The total thickness of the passivation stack was $1.2 \mu \mathrm{m}$. The PECVD depositions were done consecutively without removing the wafer from the processing chamber. In addition, the tool used had a load lock, which helps to lower the amount of contamination in the deposition chamber.

\section{RESULTS}

\section{Physical Testing}

Twenty different cantilever sizes were tested in air and water. The length and width of each cantilever was different, but they were all approximately $12 \mu \mathrm{m}$ thick. Figure 4 shows the quality factor of the first in-plane flexural mode as a function of its resonance frequency. As expected if shear forces are dominating the fluid damping, the Q-factor increases roughly with the square root of the resonance frequency. Q-factors as high as 67 were measured for a $200 \mu \mathrm{m}$ long and $90 \mu \mathrm{m}$ wide cantilever, which 
represents a considerable improvement over published data in the range of 10-20 for the out-of-plane mode in liquid [8]. As evidence of reduced mass loading effects by the surrounding fluid, the cantilevers utilizing the first in-plane flexural mode show a frequency drop of around $10 \%$ upon immersion in liquid, compared to frequency drops of the order of $50 \%$ for the out-of-plane mode.

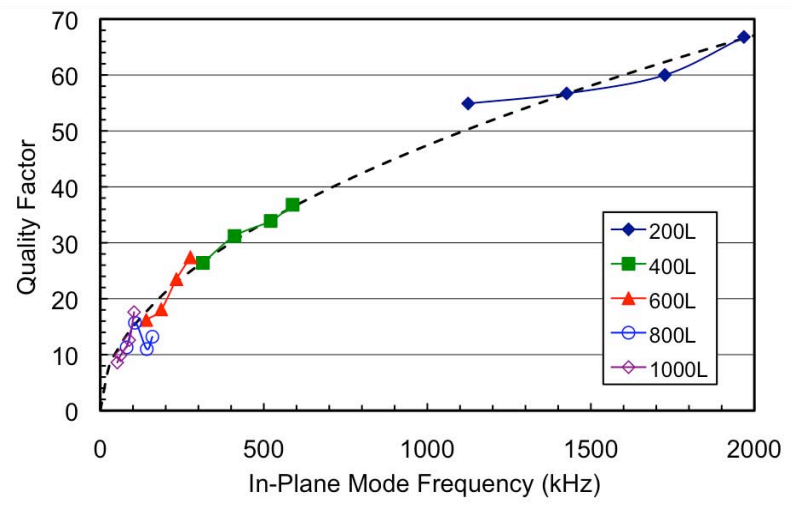

Figure 4: Quality factor in water as a function of in-plane mode frequency in water. For each length, cantilevers with a width of 45, 60, 75 and $90 \mu \mathrm{m}$ were measured. The lowest frequency in each group corresponds to the narrowest cantilever, and the frequency increases with each increasing width. The dashed line represents a square-root dependence of $Q$ with frequency.

\section{Chemical Testing}

To create mass-sensitive chemical sensors, polymer layers were deposited on $75 \mu \mathrm{m}$ wide, $400 \mu \mathrm{m}$ long, and $6.3 \mu \mathrm{m}$ thick cantilevers that also had a $3000 \AA$ gold layer on their surface (see Figure 1a). The polymer layer absorbs analyte from the environment, thus increasing the mass of the resonator. The polymers were spray-coated using an air brush on both sides of the cantilever. A $0.3 \mu \mathrm{m}$ thick PIB (polyisobutylene) film was used on each side of one cantilever, while another cantilever was coated with $2 \mu \mathrm{m}$ of EPCO (ethylene-propylene copolymer) on each side. HMDS was used as an adhesion promoter.

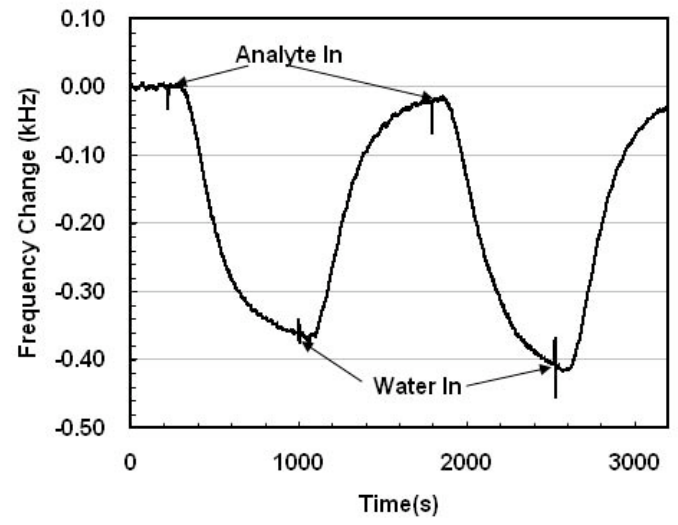

Figure 5: Response of EPCO-coated cantilever to two subsequent exposures to a 5ppm tetrachloroethylene solution. The resulting sensitivity is approx. $75 \mathrm{~Hz} / \mathrm{ppm}$. The measured short-term frequency stability is approx. $1 \mathrm{~Hz}$. The frequency spikes observed during measurement are due to pressure effects resulting from pump switching.
For testing, a system with two syringe pumps was used, with the pumps connected to the flow cell using a three-way T. PEEK tubing was used to make all connections. One syringe pump was filled with analyte solution (the analyte was kept constantly stirring for at least half an hour before use to ensure a uniform solution) another was filled with water, which was used to desorb the analyte from the polymer film.

Periodically, analyte solution was injected into the flow cell causing the frequency to decrease and then finally reach a stable level, at which point water was injected to desorb the analyte from the polymer film. As an example, Figure 5 shows the sensor response of the EPCO-coated resonator, i.e. the measured frequency change versus time for two subsequent injections of a $5 \mathrm{ppm}$ tetrachloroethylene solution. The resulting sensor sensitivity is approximately $75 \mathrm{~Hz} / \mathrm{ppm}$ at the in-plane resonance frequency of $426 \mathrm{kHz}$.

As stated above, the limit of detection (LOD) for a mass-sensitive (resonant) sensor can be estimated from

$$
\mathrm{LOD}=3 \frac{\Delta \mathrm{f}_{\min }}{\mathrm{S}}
$$

where $\mathrm{S}$ is the sensitivity in $[\mathrm{Hz} / \mathrm{ppm}]$ and $\Delta \mathrm{f}_{\min }$ is the short-term frequency stability in $[\mathrm{Hz}]$, measured in this work using the Allan variance method. Both the PIB and EPCO-coated devices tested had a frequency stability of approximately $1 \mathrm{~Hz}$ for a gate time of 4 sec. This way, the LOD of the EPCO-coated cantilever for tetrachloroethylene is estimated to be $45 \mathrm{ppb}$. Limits of detection for several other analytes dissolved in water are summarized in Figure 6 as obtained from measured sensitivity and Allan variance data using Eq. (1).

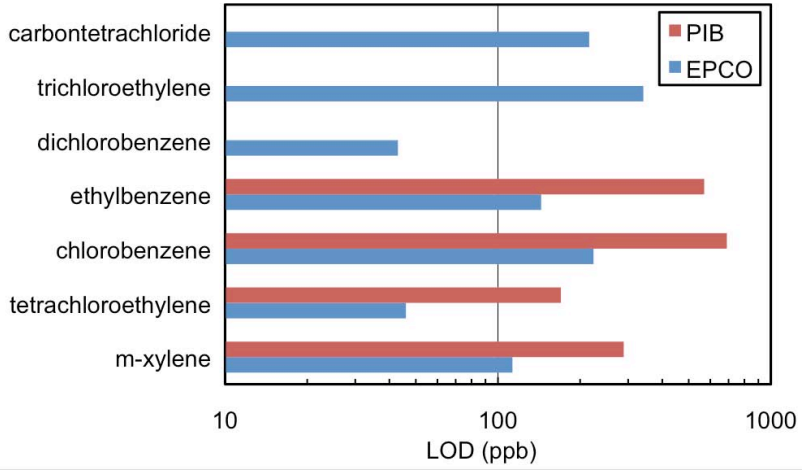

Figure 6: Limit of detection (LOD) for example analytes as obtained from experimental sensitivity and frequency stability data for $400 \mu \mathrm{m}$ by $75 \mu \mathrm{m}$ by $6.3 \mu \mathrm{m}$ cantilevers coated on both sides with either $2 \mu \mathrm{m} \mathrm{EPCO} \mathrm{or} 0.3 \mu \mathrm{m} P I B$.

The presented liquid measurements not only give low limits of detection, they were also found to be highly repeatable and consistent over several analyte concentrations. A calibration curve was constructed for tetrachloroethylene by measuring four different concentrations in duplicate using both EPCO and PIB as sensitive layers. The resulting curves with error bars (see Figure 7) show that the response of the cantilever is linear, and consistent across several different concentrations. The error bars in Figure 7 are mostly within the size of the symbols and we typically found less than $5 \%$ difference between measurements at the same concentration. 


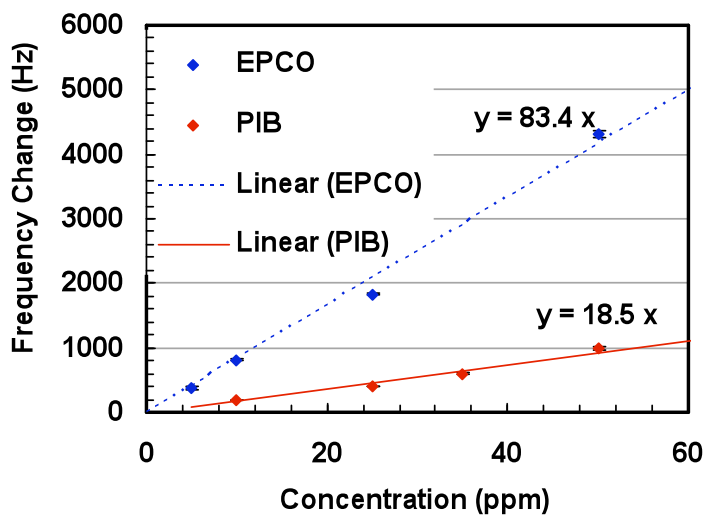

Figure 7: Measured frequency change of EPCO (blue symbols) and PIB-coated (red symbols) cantilevers as a function of the tetrachloroethylene concentration in water. The lines are linear fits to the measurement data according to the equation given in the graph.

Further improvements in the presented sensing system are possible. First, the sensitivity can be improved by using shorter cantilevers with higher resonance frequencies. Figure 8 shows simulated frequency changes for a $2 \%$ change in the density of a $4 \mu \mathrm{m}$ polymer layer on top of $12 \mu \mathrm{m}$ thick cantilevers with different dimensions. The simulations were done using eigenmode analysis in COMSOL. In combination with the results shown in Figure 4, we find that in-plane mode cantilevers with higher frequency, i.e. shorter and wider cantilevers, exhibit a higher sensitivity and a higher quality factor (and, thus, potentially better frequency stability). In addition, the effect of the cantilever thickness needs to be explored. While thinner cantilevers will yield a higher chemical sensitivity, thicker cantilevers generally exhibit higher Q-factors due to their increased inertia.

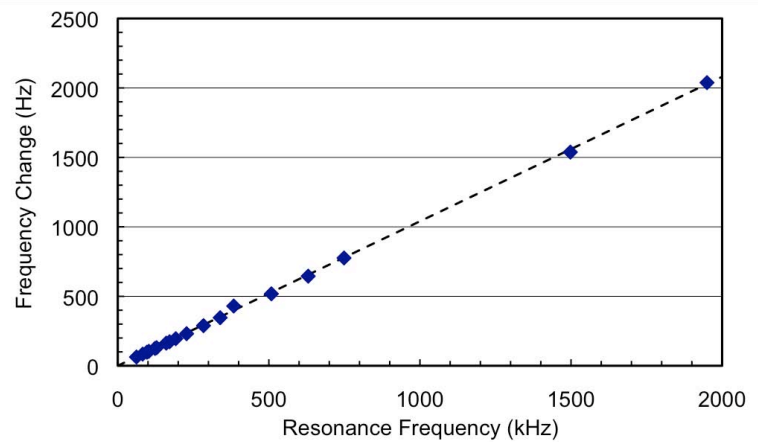

Figure 8: Simulated change of resonance frequency of polymer-coated $12 \mu \mathrm{m}$ thick silicon cantilevers to a $2 \%$ change in the polymer density as a function of the resonance frequency of the cantilever; each symbol corresponds to a cantilever with different lateral dimensions; the polymer thickness was assumed to be $4 \mu \mathrm{m}$ with a density of $840 \mathrm{~kg} / \mathrm{m}^{3}$. Using tetrachloroethylene/EPCO with a partition coefficient of $\approx 1300$ as an example, the $2 \%$ density change would stem from $a \approx 8 p p m$ analyte concentration in the surrounding liquid.

\section{CONCLUSION}

In-plane mode cantilevers were fabricated and tested as liquid chemical sensors for volatile organics in water. The devices presented here yield ppb range limits of detection for common organic pollutants in water, which approaches detection limits needed for water quality monitoring [13]. Compared to other chemical sensor approaches, the cantilevers used in this work have the advantage that their fabrication process is CMOS compatible and that they use integrated excitation and detection schemes. They can be manufactured using the same tool set and similar processing as integrated circuits, resulting in a small footprint even in array format and, thus, allowing them to be used in embedded or handheld devices.

\section{ACKNOWLEDGEMENTS}

The authors would like to thank the staff of the Georgia Tech Nanotechnology Research Center for their help. This work has been funded in part by the National Science Foundation under award ECCS-0824017.

\section{REFERENCES}

[1] G. Serrano, H. Chang, E.T. Zellers, "A micro gas chromatograph for high-speed determination of explosive vapors", Transducers and Eurosensors $15^{\text {th }}$ International Conference Solid State Actuators and Microsystems (2009), pp. 1654-1657.

[2] B. Mizaikoff, "Mid-IR fiber optic sensors", Analytical Chemistry, 75, 258A (2003).

[3] P. S. Waggoner, H. G. Craighead, "Micro- and nanomechanical sensors for environmental, chemical, and biological detection", Lab on a Chip, 7, 1238 (2007).

[4] K. M. Goeders, J. S. Colton, L. A. Bottomley, "Microcantilevers: sensing chemical interactions via mechanical motion", Chemical Reviews, 108, 522 (2008).

[5] D. Lange, C. Hagleitner, A. Hierlemann, O. Brand, H. Baltes, "Complementary metal oxide semiconductor cantilever arrays on a single chip: mass-sensitive detection of volatile organic compounds", Analytical Chemistry, 74, 3084 (2002).

[6] A. R. Krause, C. Van Neste, L. Senesac, T. Thundat, E. Finot, "Trace explosive detection using photothermal deflection spectroscopy", Journal of Applied Physics, 103, 094906 (2008).

[7] K. Naeli, O. Brand, "Dimensional considerations in achieving large quality factors for resonant silicon cantilevers in air", Journal of Applied Physics, 105, 014908 (2009).

[8] C. Vancura, Y. Li, J. Lichtenberg, K. U. Kirstein, A. Hierlemann, F. Josse, "Liquid-phase chemical and biological detection using fully integrated magnetically actuated complementary metal oxide semiconductor resonant cantilever sensor systems", Analytical Chemistry, 79, 1646 (2007).

[9] T. P. Burg, M. Godin, S. M. Knudsen, W. Shen, G. Carlson, J. S. Foster, K. Babcock, S. R. Manalis, "Weighing of biomolecules, single cells and nanoparticles in fluid", Nature, 446, 4517 (2007).

[10] Z. Li, Y. Jones, J. Hossenlopp, R. Cernosek, F. Josse, "Analysis of liquid-phase guided shear horizontal acoustic wave sensors", Analytical Chemistry, 77, 1175 (2005).

[11] J. H. Seo, O. Brand, "High Q-factor in-plane mode resonant mircrosensor platform for gaseous/liquid environment", IEEE J. Microelectromechanical Systems, 17, 483 (2008).

[12] S. Senturia, Microsystem Design, Springer, New York, 2001. [13] http://www.epa.gov/safewater/contaminants/index.html. 